NIST Special Publication 1200-11

\title{
Control experiments to avoid artifacts and misinterpretations in nanoecotoxicology testing
}

Version 1.0

Elijah J. Petersen

This publication is available free of charge from:

http://dx.doi.org/10.6028/NIST.SP.1200-11

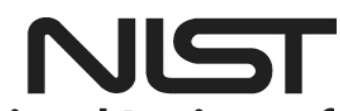




\title{
Control experiments to avoid artifacts and misinterpretations in nanoecotoxicology testing
}

Version 1.0

\author{
Elijah J. Petersen \\ Biosystems and Biomaterials Division \\ Material Measurement Laboratory
}

This publication is available free of charge from:

http://dx.doi.org/10.6028/NIST.SP.1200-11

May 2015

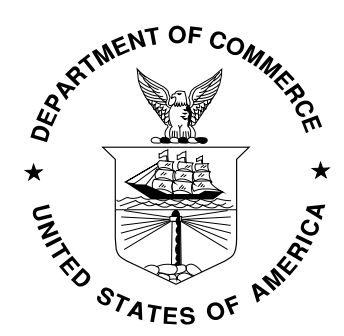

U.S. Department of Commerce

Penny Pritzker, Secretary

National Institute of Standards and Technology Willie May, Acting Under Secretary of Commerce for Standards and Technology and Acting Director 
Certain commercial entities, equipment, or materials may be identified in this document in order to describe an experimental procedure or concept adequately. Such identification is not intended to imply recommendation or endorsement by the National Institute of Standards and Technology, nor is it intended to imply that the entities, materials, or equipment are necessarily the best available for the purpose.

National Institute of Standards and Technology Special Publication 1200-11

Natl. Inst. Stand. Technol. Spec. Publ. 1200-11, 10 pages (May 2015)

CODEN: NSPUE2

This publication is available free of charge from: http://dx.doi.org/10.6028/NIST.SP.1200-11 


\section{FOREWORD}

This special publication is one in a series stemming from the National Nanotechnology Initiative (NNI) nanotechnology environmental health and safety (Nano-EHS) Research Strategy which identified Nanomaterial Measurement Infrastructure as one of the essential areas of research needed in order to develop an effective risk assessment and management plan regarding various aspects of nanotechnology in consumer products as it pertains to human health, exposure and the environment. The National Institute of Standards and Technology (NIST) was identified as a lead agency in the development of measurement strategies for the development of robust measurements to assess the potential effects of engineered nanomaterials and their fate in the environment. One important factor in these measurements is avoidance of artifacts and misinterpretations in nanoecotoxicology experiments.

The current guidance document presents potential control experiments to include during nanoecotoxicology testing to minimize artifacts and misinterpretations and to improve data reliability. Updates to this protocol may be released in the future. Visit http://nist.gov/mml/npmeasurement-protocols.cfm to check for revisions of this protocol, or new protocols in the series. We also encourage users to report citations to published work in which this protocol has been applied. 


\section{Introduction}

Making accurate measurements of the environmental fate and potential environmental and biological effects of engineered nanomaterials (ENMs) is critical for reliable risk assessment of these materials. However, results from toxicological studies, such as measurements of their cell toxicity, often differ substantially among laboratories and have questionable reliability (1-3). Moreover, numerous studies have identified artifacts (i.e., incorrect test results) during nanoecotoxicology studies as a result of the unique behaviors of ENMs (3). One important aspect of experimental design is the inclusion of control experiments to identify potential artifacts and increase reliability of the test results. Control experiments for nanoecotoxicology testing can also provide insight into the toxicity mechanism, assess dynamic changes that ENMs may undergo occur during nanoecotoxicology tests (e.g., dissolution, leaching of toxic impurities), and identify potential interferences between ENMs and the endpoint being measured. Overall, it may not be possible to accurately interpret the results from nanoecotoxicity experiments if control measurements are not included.

In this document, a number of potential control experiments for nanoecotoxicology testing, their purpose, and a list of which control experiments may be important for different experimental design considerations are described. This information is modified from a recent publication on the avoidance of artifacts and misinterpretations in nanoecotoxicology testing, and the reader is referred to that manuscript for a more complete discussion of this topic (3). While many of these same control experiments are relevant for measurements on the potential human health impacts of ENMs, the focus in this protocol is on measurements of potential ecological effects.

\section{Principles and Scope}

This protocol is prosed for the development of reliable nanoecotoxicology tests by inclusion of relevant control experiments. These control experiments are often not specific to an individual test (e.g., Daphnia magna reproduction (4)) and are considered relevant for a range of methods.

\section{Terminology}

This protocol complies with definitions relevant to nanotechnology as set forth in the ASTM International E2456 (5) and is consistent with the draft standard ISO TS 80004-1:2010 (6).

nanoparticle-sub-classification of ultrafine particle that is characterized by dimensions in the nanoscale (i.e., between approximately $1 \mathrm{~nm}$ and $100 \mathrm{~nm}$ ) in at least two dimensions; also referred to as "nano-object" in ISO TS 80004-1:2010 (6).

primary particle - the smallest discrete identifiable entity associated with a particle system; in this context, larger particle structures (e.g., aggregates and agglomerates) may be composed of primary particles.

aggregate - a discrete assemblage of primary particles strongly bonded together (i.e., fused, sintered, or metallically bonded).

Note-The adjective "primary", when used in conjunction with the term aggregate, is employed in the present context to indicate the smallest achievable dispersed particle entity. 
agglomerate-assemblage of particles (including primary particles and/or smaller aggregates) held together by relatively weak forces (e.g., van der Waals, capillary, or electrostatic), that may break apart into smaller particles upon further processing.

Note-Although we define them as distinct entities, the terms aggregate and agglomerate have often been used interchangeably to denote particle assemblies.

dispersion-used in the present context to denote a liquid (aqueous) in which particles are homogeneously suspended, or the process of creating a dispersion in which discrete particles are homogeneously distributed throughout a continuous fluid phase; implies the intention to break down agglomerates into their principal components (i.e., primary particles and/or aggregates).

\section{$\underline{4 .}$ List and description of potential control experiments}

4.1 The following are a list of potential control experiments for improving the reliability of nanoecotoxicology experiments. The purpose of the control experiments, how to conduct them, and limitations to the control experiments will be discussed.

4.2 Zero hour control: The purpose of this control is to test if ENMs cause an apparent toxicological effect (e.g., DNA damage) during processing steps after conclusion of the exposure period and if ENMs interact with test reagents or biomolecules and cause a false negative or false positive result $(7,8)$. This control is performed by adding the ENMs to the test system and then immediately testing if apparent toxicological effects are observed. For example, one cytotoxicity study spiked germanium nanoparticles to cells in media, immediately harvested the cells, and performed the Comet assay on these cells and control cells (without exposure to germanium nanoparticles) (8); they determined that there was apparent elevated DNA damage for the cells spiked with germanium nanoparticles compared to the control cells, thus indicating an artifact. One limitation of this control experiment is that ENMs may associate with the organisms or cells differently after an extended period and this could cause an artifact that would not be detected using this experiment. For example, some ENMs may enter cells and interact with the nucleus after an extended period and then be incorrectly interpreted as damaged DNA during a Comet assay measurement.

4.3 Coating control: The purpose of this control is to test if the ENM coating has toxicological or stimulatory effects on organisms or cells (9). This control is performed by testing the effect of the coating of the ENM on the ecological endpoint of interest. A certain concentration or series of concentrations of the coating should be added to the test vessel with the organisms or cells and then the ecotoxicological endpoint should be tested after the appropriate exposure duration. If only a single concentration is tested, the concentration of the coating in the ENM dispersion for the highest ENM concentration should be tested. If a series of concentrations are tested which can be used to provide a dose-response curve, this series should include the highest coating concentration tested. One limitation of this test is that it may be challenging to relate results from the coating control experiment to those from the ENM ecotoxicity experiment. Only a small fraction of the ENM coating may be released from the ENM during an ecotoxicological experiment and thus testing the total coating mass on the organism may overestimate the toxicological or stimulatory impact. 
4.4 Direct interference control (production of a signal similar to measurand): The purpose of this control is to assess if ENMs produce a signal (e.g., absorbance, fluorescence) that could impact the analytical method (7). This control is performed by adding ENMs to test containers without organisms or cells and then assessing if the ENMs produce a sufficiently high signal to impact the measurand. This control can also be performed by adding the ENMs to test containers without organisms or cells for the duration of the experiment and then assessing if the ENM would impact the measurand. One limitation for this test is that the ENMs may undergo different dynamic changes (e.g., rate of agglomeration) in the test media during incubation with organisms or cells. The different changes to the ENMs in the presence of organisms or cells in comparison to the ENM changes in a test container without organisms or cells could influence the potential for the ENM to interfere with the assay. For example, passage through the gut tract of an organism could lead to enhanced agglomeration, or association with cells could influence an assay that tests a property (i.e., absorbance) of the cells.

4.5 Dispersant control: The purpose of this control is to test if the dispersant has toxicological or stimulatory effects on organisms or cells $(10,11)$. This control is performed by testing the effect of a dispersant on the ecological endpoint of interest. A certain concentration of the dispersant should be added to the test vessel with the organism or cells and then the ecotoxicological endpoint should be tested after the appropriate exposure duration. Similarly to the limitations for the coating control experiment, it may be challenging to determine what concentration of dispersant to test.

4.6 Dissolved ion control: The purpose of this control is to compare toxicological endpoint results between ENMs and their constituent dissolved ions and to assess if nanoparticle formation could occur from ions while in the test media, organisms, or cells $(12,13)$. This control is performed by testing the ecotoxicological effects of dissolved ions at similar concentrations to those of the ENMs. If the ENMs have a greater effect than would occur for the concentration of dissolved ions released from the ENMs under these experimental conditions, then there may be an ENM-specific ecotoxicological effect. One limitation of this test is that nanoparticles may be formed from ions under certain conditions (i.e., silver nanoparticle formation) and thus the organisms in the ion treatment condition could be exposed to a combination of ions and nanoparticles. Overall, careful characterization of the test media is critical.

4.7 Endotoxin inhibition/enhancement control: The purpose of this control is to assess if there is an impact from the presence of ENMs on effects caused by endotoxins for a specific endpoint (14). This control is performed by assessing if the presence of ENMs at the test concentrations for the assay duration influences the effects of endotoxins either by increasing or decreasing endotoxin effects (e.g., inflammation). One limitation of this control is that endotoxins are highly variable in composition. Thus, the composition of endotoxins purchased from a commercial source and added during this control experiment may differ from those inadvertently associated with the ENMs from, for example, the ENM synthesis process.

4.8 Filtrate only control: The purpose of this control is to assess potential toxicity of contaminants on and dissolution from ENMs from the synthesis, storage, and dispersion processes (9). This control is performed by preparing a dispersion with the ENMs, filtering the 
samples using a filter setup that is sufficient to remove the ENMs, and then testing the potential ecotoxicological effects of the remaining filtrate. It should be noted though that the filtration apparatus may adsorb some fraction of the contaminants or dissolved ions, and thus, this control may underestimate the impact of toxins present other than the ENMs.

4.9 Larger/bulk particle control: The purpose of this control is to compare endpoints with ENMs and larger/bulk particles of a similar elemental composition to assess if nanoparticle-specific effects are observed (12). This control is performed by testing the ecotoxicological effects of larger/bulk particles at a similar concentration or range of concentrations for dose-response measurements as those of the ENMs. If the ENMs have a greater effect than larger/bulk particles, then there may be an ENM-specific ecotoxicological effect. One limitation of this control is that larger/bulk particles are rarely identical to the ENMs in all properties except size (15). For example, Degussa $\mathrm{P} 25 \mathrm{TiO}_{2}$ is a combination of anatase and rutile and it is challenging to find bulk particles with a similar ratio of $\mathrm{TiO}_{2}$ particle crystal structures. Thus, it is often not possible to only change the particle size while retaining all other relevant characteristics. In addition, there are not straightforward bulk particles for comparing the size effects of carbon nanomaterials.

4.11 Nutrient depletion control: The purpose of this control is to assess the extent to which adsorption of media constituents by ENMs could have an indirect (toxic) effect (16). This control can be assessed by adding ENMs to the test media, incubating for a time interval similar to that of the ecotoxicological test, removing the ENMs such as with filtration, and then testing if there is a change in the ecotoxicological endpoint with this media. An alternative approach is to quantify the concentration of critical elements in the tissue of the organism after the exposure period to test if there is a decrease compared to the control organisms. A third approach is to perform a sorption experiment to assess the extent of sorption of the test media components onto the ENM, prepare test media with lower concentrations of the test media components based on the decrease caused by removal of the components from sorption onto the ENM, and then test if there is a change in the ecotoxicological endpoint with this modified media. One limitation to testing effects of nutrient depleted media is that there may be desorption of nutrients from the ENMs during passage through the gut tract of organisms and thus the depleted media might overestimate the toxicological impact of nutrient depletion.

4.12 Shading control: The purpose of this control is to assess the extent to which light intensity reduction caused by ENMs could impact the ecotoxicity endpoint measured $(17,18)$. The first step is to analyze the decrease in the transmittance through the test container containing the ENM from the specified light source; this could be conducted at the highest ENM concentration or at a range of ENM concentrations if a dose-response relationship is being tested. The second step is conduct a "shading" control experiment by running the toxicity test without ENMs but with a decreased light transmittance(s) identical to the decreased light transmittance from the ENM concentration(s) measured in the first step. The third step is to compare the results from the toxicity endpoint between the "shading" control experiment and control measurements (without ENMs and without decreased light transmittance) and measurements with ENMs. If the assay results for the control experiments with and without "shading" are similar, this finding suggests that shading effects from the ENM are not impacting the assay result. If the assay results from the control experiment with "shading" are similar to the results from tests with 
ENMs, the ecotoxicity effect of the ENMs may be largely explained by a "shading" effect. One limitation of this control is that there may be localized shading if ENM agglomerates are directly associated with, for example, algal cells. This control may underestimate the impact of reduced light intensity from shading in that situation.

4.13 Sonication control with media and organic chemicals/coatings: The purpose of this control is to investigate possible changes to media constituents or toxicological properties of organic chemicals from sonication (19). This control can be tested by adding the test media and any organic chemicals in the test media (e.g., cholesterol), dispersants, or coatings to the container used to sonicate samples, sonicating this sample using the same conditions as those that will be used to disperse the ENMs, and then assessing if this solution would impact the ecotoxicological endpoint being studied. One limitation to this approach is that the presence of ENMs may influence the degree of damage to media constituents during ultrasonication. For example, a recent study found reduced oxidative damage to DNA oligomers during sonication when singlewall carbon nanotubes were present (20).

\section{$\underline{5 . \text { When to use various control experiments }}$}

5.1 The following is a list of potential situations when including various control experiments described above in the experimental design may be beneficial. The choice of when to use which control experiments will also depend on expert judgement as many of them may not be relevant for any particular study.

5.2 If it is important to assess if toxic impurities on the ENMs, coatings, or dispersants are impacting the nanoecotoxicology result, it may be helpful to include a coating control, dispersant control, endotoxin inhibition/enhancement control, a filtrate-only control, and a sonication control with media and organic chemicals/coatings in the experimental design.

5.3 If it is important to assess if there is a nanoparticle-specific effect being observed, it may be helpful to include a dissolved ion control and a larger/bulk particle control in the experimental design.

5.4 If it is important to assess if the ENMs may be unexpectedly causing a false positive or false negative result, it may be helpful to conduct a zero hour control and a direct interference control.

5.5 If it is important to understand the mechanism of the toxic effects observed, it may be helpful to include a coating control, dispersant control, dissolved ion control, endotoxin enhancement/inhibition control, filtrate only control, larger/bulk particle control, nutrient depletion control, shading control, and sonication control.

\section{Abbreviations}

ENM

engineered nanomaterial 


\section{Acknowledgements}

I acknowledge all of my coauthors involved in the writing of the review paper from which this protocol was adapted.

\section{References}

1. Krug, H. F.; Wick, P. Angewandte Chemie (International ed in English) 2011, 50 (6), 1260-1278.

2. $\quad$ Schrurs, F.; Lison, D. Nat Nano 2012, 7 (9), 546-548.

3. $\quad$ Petersen, E. J.; Henry, T. B.; Zhao, J.; MacCuspie, R. I.; Kirschling, T. L.;

Dobrovolskaia, M. A.; Hackley, V.; Xing, B.; White, J. C. Environ. Sci. Technol. 2014, 48 (8), 4226-4246.

4. Organization for Economic Cooperation and Development. 1998. Daphnia magna reproduction test - Guideline 211. Paris, F.

5. ASTM (American Society for Testing Materials) International, E2456-06: standard terminology relating to nanotechnology. West Conshohocken, PA., 2006.

6. ISO (International Organization for Standardization), TS 80004-1: nanotechnologies vocabulary - Part 1: Core terms. Geneva, Switzerland, 2010.

7. $\quad$ Horst, A. M.; Vukanti, R.; Priester, J. H.; Holden, P. A. Small 2013, 9 (9-10), 1753-1764.

8. $\quad$ Lin, M. H.; Hsu, T. S.; Yang, P. M.; Tsai, M. Y.; Perng, T. P.; Lin, L. Y. Inter. J. Rad. Biol. 2009, 85 (3), 214-226.

9. $\quad$ Petersen, E. J.; Pinto, R. A.; Mai, D. J.; Landrum, P. F.; Weber, W. J., Jr. Environ. Sci. Technol. 2011, 45 (3), 1133-1138.

10. Gao, J.; Llaneza, V.; Youn, S.; Silvera-Batista, C. A.; Ziegler, K. J.; Bonzongo, J. C. J. Environ. Toxicol. Chem. 2012, 31 (1), 210-214.

11. Youn, S.; Wang, R.; Gao, J.; Hovespyan, A.; Ziegler, K. J.; Bonzongo, J. C. J.; Bitton, G. Nanotoxicology 2012, 6 (2), 161-172.

12. Atha, D. H.; Wang, H. H.; Petersen, E. J.; Cleveland, D.; Holbrook, R. D.; Jaruga, P.; Dizdaroglu, M.; Xing, B. S.; Nelson, B. C. Environ. Sci. Technol. 2012, 46 (3), 1819-1827.

13. Scanlan, L. D.; Reed, R. B.; Loguinov, A. V.; Antczak, P.; Tagmount, A.; Aloni, S.; Nowinski, D. T.; Luong, P.; Tran, C.; Karunaratne, N.; Pham, D.; Lin, X. X.; Falciani, F.; Higgins, C. P.; Ranville, J. F.; Vulpe, C. D.; Gilbert, B. Acs Nano 2013, 7 (12), 10681-10694. 14. Dobrovolskaia, M. A.; McNeil, S. E., Nanoparticles and endotoxin. In Handbook of immunological properties of engineered nanomaterials, World Scientific Publishing: Singapore, 2013; pp 77-110.

15. Handy, R. D.; van den Brink, N.; Chappell, M.; Muhling, M.; Behra, R.; Dusinska, M.; Simpson, P.; Ahtiainen, J.; Jha, A. N.; Seiter, J.; Bednar, A.; Kennedy, A.; Fernandes, T. F.; Riediker, M. Ecotoxicology 2012, 21 (4), 933-72.

16. Zhao, X.; Ong, K. J.; Ede, J. D.; Stafford, J. L.; Ng, K. W.; Goss, G. G.; Loo, S. C. J. Small 2013, 9 (9-10), 1734-1741.

17. Schwab, F.; Bucheli, T. D.; Lukhele, L. P.; Magrez, A.; Nowack, B.; Sigg, L.; Knauer, K. Environ. Sci. Technol. 2011, 45 (14), 6136-6144.

18. Long, Z. F.; Ji, J.; Yang, K.; Lin, D. H.; Wu, F. C. Environ. Sci. Technol. 2012, 46 (15), 8458-8466. 
19. Wang, R.; Hughes, T.; Beck, S.; Vakil, S.; Li, S.; Pantano, P.; Draper, R. K. Nanotoxicology 2013, 7 (7), 1272-1281.

20. Petersen, E. J.; Tu, X. M.; Dizdaroglu, M.; Zheng, M.; Nelson, B. C. Small 2013, 9 (2), 205-208. 